\title{
Stabilization of $\gamma$-sterilized low-density polyethylene by synergistic mixtures of food-contact approval stabilizers
}

\author{
Sameh A. S. Alariqi ${ }^{1}$ • Niyazi A. S. Al-Areqi ${ }^{1} \cdot$ Elyas Sadeq Alaghbari $^{1}$ • \\ R. P. Singh ${ }^{2}$
}

Received: 7 October 2015/Accepted: 17 March 2017/Published online: 31 March 2017

(c) The Author(s) 2017. This article is an open access publication

\begin{abstract}
In our previous studies, we have found the synergistic combinations of stabilizers which follow different mechanisms of stabilization and are approved for food contact applications. The present attempt is to test the potentials of those systems in stabilizing $\gamma$-sterilized lowdensity polyethylene (LDPE). The results were discussed by comparing the stabilizing efficiency of mixtures with and without phenol systems as well as with their counterparts of isotactic polypropylene (iPP) and ethylene-propylene copolymers (EP) matrices. LDPE has been melt-mixed with tertiary hindered amine stabilizer (tert-HAS), oligomeric HAS stabilizer, phenolic and organo-phosphite antioxidants and subjected to $\gamma$-sterilization. Stabilization in terms of changes in oxidation products, tensile properties, yellowing and surface morphology was evaluated by FT-IR spectroscopy, Instron, colorimetry, and scanning electron microscopy (SEM), respectively. The results of the present study confirm the validity of those systems for protecting various polyolefins against $\gamma$-sterilization. The results showed that the synergism, antagonism and the trend in stabilization efficiency of the binary, ternary and quaternary stabilizer systems were almost similar in LDPE, iPP and EP matrices. The binary system of oligomeric HAS and tert-HAS has shown the antagonistic effect of
\end{abstract}

Sameh A. S. Alariqi

samehalariqi@yahoo.com

R. P. Singh

rp.singh@ncl.res.in

1 Department of Chemistry, Faculty of Applied Science, University of Taiz, P. O. Box: 4007, Taiz, Yemen

2 Division of Polymer Science and Engineering, National Chemical Laboratory, Dr. Homi Bhabha Road, Pune 411008, India stabilization, whereas their combination with organophosphite has exhibited synergistic effect even at higher doses of $\gamma$-sterilization. The combination of oligomeric HAS, tert-HAS, organo-phosphite and hindered phenol exhibited improved stabilization efficiency than single or binary additive systems. The phenol systems have shown long term of stability than that of phenol-free systems. It was found that the consumption of oligomeric stabilizer significantly depends on the components of stabilization mixture. It was concluded that the stability of polyolefins (LDPE, iPP and EP) against $\gamma$-sterilization can be achieved by blends of different stabilizers which are approved for food contact applications.

Keywords LDPE $\cdot \gamma$-Sterilization · Stabilization · Oligomeric HAS stabilizer - Synergistic mixtures

\section{Introduction}

LDPE is one of the most popular polymers in the manufacturing of food packaging and medical disposables, because it exhibits high transparency, good mechanical properties, low cost, good sealability and chemical resistance, and can be employed over a wide temperature range. Treatment with gamma radiation is becoming a common process for the sterilization of food packaging and medical plastics. The most commonly validated dose used for sterilization is $25 \mathrm{kGy}$ [1]. However, using $\gamma$-radiation for sterilization of packaging and medical plastics is known to result in physical changes, including embrittlement, stiffening, softening, discoloration, odor generation and a decrease in molecular weight [2-5]. The degradation of sterilized plastics continues for a long time during their shelf life and service, which is called post-degradation or 
post-sterilization. Radiation-induced changes in the physical properties of a packaging material and medical plastics should not impair its function and the degradation products as well as the utilized additives should be non-toxic [6]. The radiation stability of polyolefins can be done at different stages of degradation process by adding very small amounts of additives $(0.05-0.5 \% \mathrm{w} / \mathrm{w})$ called as 'stabilizers'. They are radical scavengers, antioxidants and hydroperoxide decomposers which follow different action mechanism. Antioxidants are incorporated in the polymer formulation to inhibit the attack of oxygen during the processing and $\gamma$-sterilization of the polymer [7]. Phenols, phosphites, or amine compounds are used as antioxidants depending on the free radicals expected to form. Phenolic antioxidants (Primary antioxidants) are generally radical scavengers or H-donors such as Irganox-1010. They are extremely effective at preserving physical properties of polymer during and after $\gamma$-sterilization, but at the expense of yellow color formation [8]. Consequently, antioxidants such as hindered phenols are unacceptable medically and for the food packaging because of the intense yellow discoloration which results from the formation of compounds such as stibenequinones upon $\gamma$-sterilization. Secondary antioxidants (organo-phosphites) are typically hydroperoxide decomposers (i.e. Irgafos-168) inhibiting oxidation by decomposing the hydroperoxides to form stable products. Unlike primary antioxidants, secondary antioxidants are inadequate if they used alone, so they are usually used in combination with primary antioxidants to get synergistic effects [9]. An organo-phosphite may be used as a shortterm antioxidant to protect the polymer during processing, while phenolic antioxidants are used for long-term protection. Hindered amine stabilizers (HAS) are widely used radical scavengers having multifunctional capabilities for scavenging radicals. A substituted piperidine was found to give good protection (little yellowing or embrittlement) against $\gamma$-irradiation as well as post-irradiation storage under accelerated test conditions $\left(60^{\circ} \mathrm{C}\right.$ in air) [10]. The efficiency of stabilizers is very much dependent upon the type and the grade of polymer in which they are compounded; thus the judicious selection of stabilizers is very important in the formulation of plastic [11]. These additives are not chemically bound to the polymer matrix and migrate or leach out under the influence of physicochemical factors such as temperature, sterilization and type of solvents and $\mathrm{pH}$ of the packaged product [12]. Unfortunately, the toxicological data on most of the stabilizers are either not available or incomplete and for many antioxidants are available from feeding studies only [7]. Polymer stabilization is a dynamic process resulting in many transformed and degradation products which are potentially leachable and extractable [12]. Many antioxidants and stabilizers act sacrificially and are converted to oxidation products during the process of stabilization [13]. In fact, there is a little knowledge regarding the toxicity of antioxidant transformation products; thus there is a doubt that they may be more toxic than the antioxidants from which they are derived [13]. It is urged that when more than one stabilizer is utilized, toxicity must be estimated by considering the combination rather than each agent alone, since a different synergistic effect could be the result very often [14]. Consequently, the toxicology of food packaging and medical plastics depends on many factors such as the effect of stabilizer loss, toxicity of migrated or leached stabilizers, degradation process and the degradation products and the effect of sterilization methods on the plastics or its constituents [7]. Migration and leachability of nonpolymeric components to its environment (esp. into drugs, body fluids and foods) gives rise to major concerns in case of food packaging plastics, packaging materials for pharmaceuticals and other medical applications. This migration is associated with health hazards and has become a major factor in regulations regarding the safety and quality of packaged food. Thus, stabilization should be done with stabilizers which are approved for food contact and biomedical applications.

It is well known that the efficiency of stabilizers is disturbed by a loss of the active form of stabilizers. This loss can be either chemical consumption or physical loss. The consumption of the stabilizers occurs during chemical reactions in the presence of light, heat and radiation. However, the physical loss of the stabilizers occurs by diffusion toward the polymer surface by evaporation, volatilization, poor solubility, leachability and migration into the material in contact with the polymer $[15,16]$. The consumption and loss of the stabilizers accelerate the aging of the polymer more than thermal- or radio- or photo-oxidation $[17,18]$. The long-term protection was observed with oligomeric HAS stabilizer, whereas very short-time protection was found with low-molecular weight HAS [19-22]. Polymeric hindered amine light stabilizers (HALS) shows a much higher thermal stability and better extraction resistance than that of low molecular weight; thus, the tendency for developing amine stabilizers in the form of oligomeric/polymeric macromolecules recently established [23, 24]. HALS was developed from lowmolecular-weight stabilizers to high molecular weight to counteract the effects of volatilization and extraction from the polymer matrix during outdoor application [25, 26]. The low-molecular-weight stabilizers mostly are liquid, volatile and easily decompose in thermal processing temperatures; thus the effective concentration of such admixture in the polymer is reduced. On the other hand, the lowmolecular-weight stabilizer has good mobility and usually it can be dispersed more homogeneously in the polymeric materials than the high-molecular-weight stabilizer [23]. It 
was concluded that the stabilizer mobility played an important role in the overall mechanism of stabilization of HAS in PP [27]. It was reported that approximately $95 \%$ molecules of oligomeric stabilizer were translationally immobile in the polymer matrix and it was explained that decreased efficiency of oligomeric stabilizers with increased molecular weight was a result of reduced stabilizer mobility [28]. Gugumus [29] determined the optimum molecular weight (MW) to be about 2700 for poly (1,2,2,6,6-pentamethyl-4-piperidyl acrylate) for the light stabilization of PP. Thus, there have been always some disadvantages in using single-additive system such as compatibility, migration with low-molecular-weight stabilizers (especially HAS), immobility with high-molecularweight stabilizers, yellowing with phenolic antioxidants and reduced efficiency of organo-phosphites by fast consumption.

Considering the above said aspects, we have found the combinations of synergistic mixtures of oligomeric stabilizers in our previous works [20-22] where we have used mixtures of oligomeric HAS and tert-HAS, primary and secondary antioxidants, which are approved for food contact applications [30], and their selection has been based on different molecular weights and protecting mechanisms in iPP and EP matrices. The previous work aimed to comprise heterosynergistic combinations of these stabilizers to be an alternative stabilization system of the phenolic antioxidants as well as to improve the discoloration of the phenolic system. Two groups of stabilization systems were prepared and tested on iPP and EP matrices upon $\gamma$-sterilization. The first group was phenol-free system where phenol is excluded due to its discoloration disadvantage and the second group was a phenolic system where the phenol is blended with other stabilizers to improve its discoloration. The binary (1:1) phenol-free system (oligomeric HASorgano-phosphite) and binary (1:1) phenolic system (oligomeric HAS-phenol antioxidant), ternary (1:1:1) phenolfree system (oligomeric HAS-tertiary HAS-organo-phosphite) and ternary (1:1:1) phenolic system (oligomeric HAS-phenol antioxidant-organo-phosphite) and quaternary (1:1:1:1) system of all four stabilizers has shown improved stability against $\gamma$-sterilization. However, the antagonistic effect of stabilization was found in the binary (1:1) phenol-free system (oligomeric HAS-tert-HAS). Stabilization and synergistic mechanisms as well as the reactivity of the products resulted due to the combination of those stabilizers have been discussed in our previous work [20-22]. Since the stabilizer blend systems we tested are still new (especially with $\gamma$-irradiation), there are no clear data on the toxicity of the formed products. The aim of our research series was to improve the stability of polyolefins against $\gamma$-sterilization by preparing synergistic mixtures based on oligomeric stabilizer using food-approved stabilizers. Since we tested those stabilization systems on iPP and EP matrices it will be beneficial to test their effectiveness on PE matrix and to study the validity of those systems for protecting different polyolefins against $\gamma$ sterilization. Thus, it will be worthwhile for making a vast array of data on stabilization of food packaging and biomedical plastics, to study stabilizing efficiency of those systems with LDPE, which are widely desired as food packaging and medical plastic. The major objective of this work was to conduct a comparative study on the effect of those stabilizing systems on LDPE with other polyolefin matrices (iPP and EP copolymers).

\section{Experimental method}

\section{Materials and chemicals}

A commercial sample of LDPE (density: $0.92 \mathrm{~g} / \mathrm{cm}^{3}$, Melt flow index (MFI): $1.2 \mathrm{~g} / 10 \mathrm{~min}$ ) was obtained from Indian Petrochemicals Corp Ltd., India, under the trade name INDOTHENE. The LDPE was purified as follows: pellets of LDPE were dissolved in xylene by gentle heating under reflux under a nitrogen atmosphere for $1 \mathrm{~h}$. Addition of cold methanol caused a precipitate to form. This was filtered and then dried at $50{ }^{\circ} \mathrm{C}$ in a vacuum oven until constant weight. The sample was assumed to be "additive free" and designated as purified sample. Solvents were obtained from M/s. SD. Fine Chemicals Ltd, Mumbai, India. Four different stabilizers supplied by M/s. CibaGeigy, Switzerland, which is approved for food contact applications [30], were used in this study and they are as follows:

1. Tinuvin 765 (CAS No.: 41556-26-7 and 82919-37-7), a low molecular weight $(\mathrm{MW}=508 \mathrm{~g} / \mathrm{mol})$, tertiary HAS, yellow liquid, designated as (T),

2. Chimassorb 944 (CAS No.:71878-19-8), an oligomeric HAS, high molecular weight $(\mathrm{MW}=2790 \mathrm{~g} / \mathrm{mol}$, $M_{\mathrm{n}} \approx 3000$ ), secondary HAS, white powder, mp $115-125^{\circ} \mathrm{C}$, designated as (C),

3. Irganox 1010 (CAS No.: 6683-19-8), a hindered phenol $(\mathrm{MW}=1178 \mathrm{~g} / \mathrm{mol})$ designated as $(\mathrm{X})$ and

4. Irgafos 168 (CAS No.: 31570-04-4), an organo-phosphite, IV (MW = $649.9 \mathrm{~g} / \mathrm{mol}$ ) designated as (S).

The chemical structure and IUPAC name of the stabilizers are given below: 


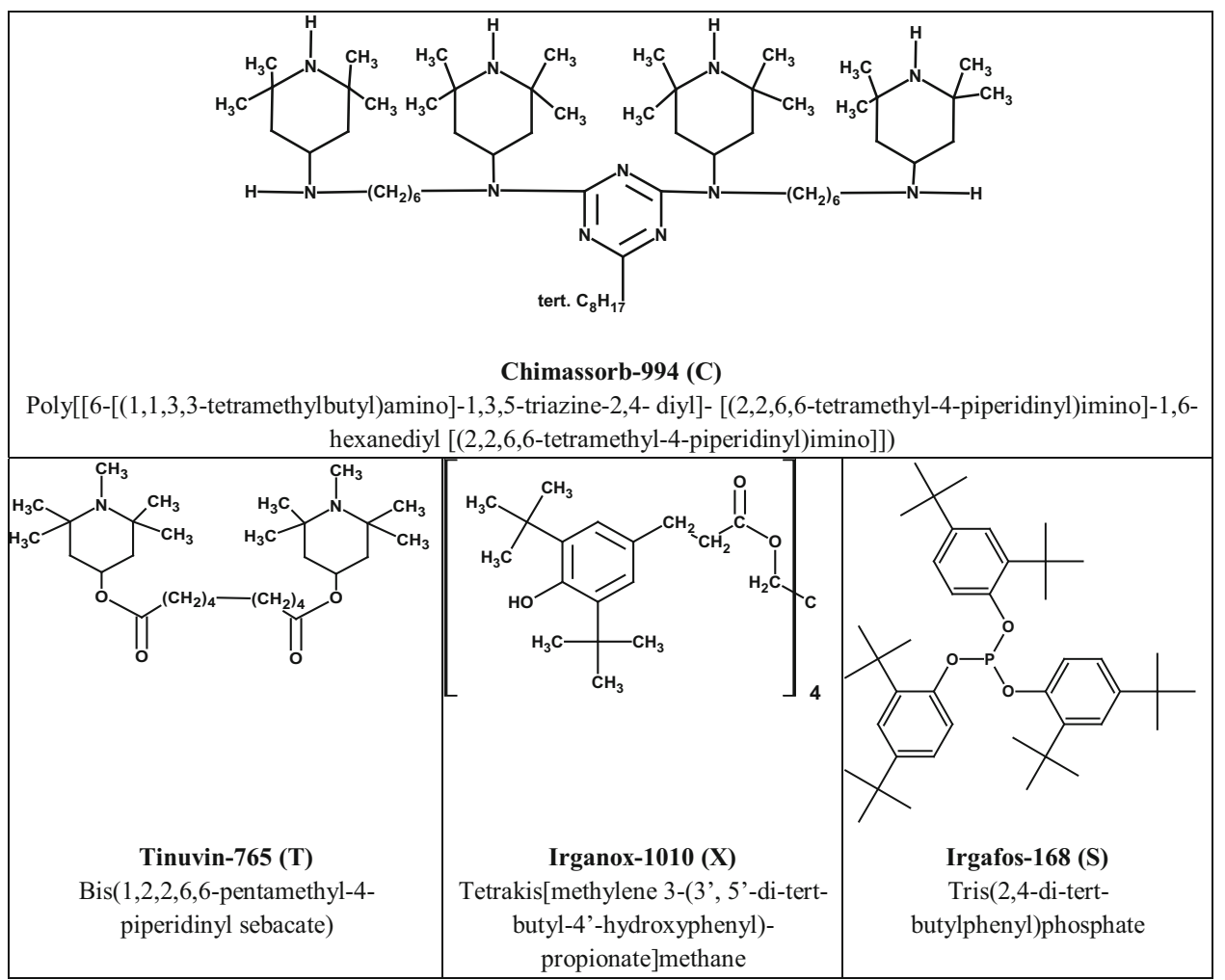

\section{Mixing of stabilizers and preparation of specimens}

The weighed amount of stabilizers was dissolved in chloroform and mixed with the required amount of dried polymer powder (LDPE) for better distribution and chloroform was evaporated and dried at $50{ }^{\circ} \mathrm{C}$ in vacuum oven. After drying, this polymer was meltmixed in a microcompounder (DSM, The Netherlands) for $5 \mathrm{~min}$ at $160{ }^{\circ} \mathrm{C}$. Keeping the ratio between polymer and stabilizer for each blend system as 99.6 polymer: $0.4 \%(\mathrm{w} / \mathrm{w})$ stabilizers $(9.6 \mathrm{~g}$ polymer: $0.4 \mathrm{~g}$ stabilizer), and the ratio between the stabilizers as $1: 1$ for binary (9.6 g polymer: $0.2 \mathrm{~g}$ for each stabilizer), $1: 1: 1$ for ternary ( $9.6 \mathrm{~g}$ polymer: $0.133 \mathrm{~g}$ for each stabilizer) and 1:1:1:1 for quaternary blend systems (9.6 $\mathrm{g}$ polymer: $0.1 \mathrm{~g}$ for each stabilizer) as tabulated in Tables 1 and 2, the samples were compounded. Then, they were molded as films in aluminum foil between two plates by heating up to $160{ }^{\circ} \mathrm{C}$ and holding for $3-5 \mathrm{~min}$ and then increasing the molding pressure to $15,000 \mathrm{lb}$. The pressure was allowed to fall, and the molds were then immediately quenched into a large bath filled with water at $20{ }^{\circ} \mathrm{C}$. Their thickness was found to be about $100 \pm 10 \mu \mathrm{m}$.

\section{$\gamma$-Irradiation}

The films were kept in a cylindrical well-type ${ }^{60} \mathrm{Co} \gamma$-irradiation chamber (Made by Bhabha Atomic Research Centre, Bombay, India) in the position which allows a uniform irradiation for all films. The samples were irradiated at different doses: 25 (sterilization dose), 50, 75 and

Table 1 Phenol-free system

\begin{tabular}{|c|c|c|c|c|c|c|c|}
\hline \multirow[t]{2}{*}{ S. no } & \multirow[t]{2}{*}{ Sample code } & \multicolumn{2}{|l|}{ Polymer } & \multicolumn{4}{|c|}{ Stabilizers $\%(\mathrm{w} / \mathrm{w})$ or $\mathrm{g}$} \\
\hline & & $\%(\mathrm{w} / \mathrm{w})$ & G & Irganox-1010 & Chimassorb-944 & Tinuvin-765 & Irgafos-168 \\
\hline 1 & $\mathrm{C}$ & 99.6 & 9.6 & - & 0.4 & - & - \\
\hline 2 & $\mathrm{~T}$ & & & - & - & 0.4 & - \\
\hline 3 & $\mathrm{~S}$ & & & - & - & - & 0.4 \\
\hline 4 & CT & & & - & 0.2 & 0.2 & - \\
\hline 5 & $\mathrm{CS}$ & & & - & 0.2 & & 0.2 \\
\hline 6 & CTS & & & - & 0.133 & 0.133 & 0.133 \\
\hline
\end{tabular}


Table 2 Phenol System

\begin{tabular}{|c|c|c|c|c|c|c|c|}
\hline \multirow[t]{2}{*}{ S. no. } & \multirow[t]{2}{*}{ Sample code } & \multicolumn{2}{|l|}{ Polymer } & \multicolumn{4}{|c|}{ Stabilizers $\%(\mathrm{w} / \mathrm{w})$ or $\mathrm{g}$} \\
\hline & & $\%(\mathrm{w} / \mathrm{w})$ & G & Irganox-1010 & Chimassorb-944 & Tinuvin-765 & Irgafos- 168 \\
\hline 7 & $\mathrm{X}$ & 99.6 & 9.6 & 0.4 & - & - & - \\
\hline 8 & $\mathrm{CX}$ & & & 0.2 & 0.2 & - & - \\
\hline 9 & CTX & & & 0.133 & 0.133 & 0.133 & - \\
\hline 10 & CXS & & & 0.133 & 0.133 & - & 0.133 \\
\hline 11 & CTXS & & & 0.1 & 0.1 & 0.1 & 0.1 \\
\hline
\end{tabular}

$100 \mathrm{KGy}$ (dose rate $0.4 \mathrm{kGy} \mathrm{h}^{-1}$ ) at room temperature in air. Irradiation experiments were performed at the Nuclear Chemistry Department, University of Pune, India.

\section{Characterization}

\section{FT-IR spectroscopy}

FT-IR spectroscopy (Perkin Elmer 16 PC FT-IR spectrophotometer) was used to characterize the chemical changes caused by $\gamma$-radiation in the polymer specimens. Oxidation products were identified and quantified and our interest was mainly focused on the changes in the carbonyl region $\left(1600-1800 \mathrm{~cm}^{-1}\right)$ to follow $\gamma$-induced oxidation. The IR spectrometer was used to measure the concentration of carbonyl compounds in the polymer specimens at $1720 \mathrm{~cm}^{-1}$. A value of $220 \mathrm{~L} \mathrm{~mol}^{-1} \mathrm{~cm}^{-1}$ was used for absorption coefficient [31]. The spectrometer was operated at a resolution of $4 \mathrm{~cm}^{-1}$. The oxidized specimens were analyzed immediately to minimize the post $\gamma$-effect.

\section{Universal testing machine}

The changes in the mechanical properties were measured by a universal testing machine (Instron model 4201, Instron, MA, USA). Elongation at break was determined from stress-strain curves. The cross speed used was $10 \mathrm{~mm} \mathrm{~min}^{-1}$. The specimens were cut according to IS: 2808-1984:A4, (100 mm length, $10 \mathrm{~mm}$ width and the gauge space $50 \mathrm{~mm}$ ). The results of each sample were taken as the average of five specimens.

\section{Color measurements}

Yellowness index (YI) was determined in accordance with ASTM D1925 [32] by reflectance measurements using a Color Mate HDS Colorimeter (Milton Roy, USA) with integrating sphere. The samples were placed in the reflectance part of a sphere using a standard white ceramic as reference tile. The instrument is designed to give direct yellowness index value on the basis of CIE standard illumination C (CIE 1931) $2^{\circ}$ standard observer viewing [33]. It was obtained from the tristimulus values $X_{\mathrm{CIE}}, Y_{\mathrm{CIE}}$ and
$Z_{\mathrm{CIE}}$ relative to source $\mathrm{C}$ using the equation $\mathrm{YI}=[100$ $\left.\left(1.28 X_{\mathrm{CIE}}-1.06 Z_{\mathrm{CIE}}\right)\right] / Y_{\mathrm{CIE}}$. Several values of $\mathrm{YI}$ obtained from different parts of the samples were generally used to obtain an average value of the yellowness index. The yellowness index represented in terms of delta yellowness index $(d$ YI):

Delta yellowness index $(d$ YI)

$=$ Yellow index after $\gamma$-irradiation

- Yellow index before $\gamma$-irradiation.

\section{Scanning electron microscopy}

Fracture surface produced by subjecting specimens to $\gamma$ radiation was determined by scanning electron microscopy. The stained samples were dried under vacuum for $24 \mathrm{~h}$ at $50{ }^{\circ} \mathrm{C}$. These gold-coated samples were scanned under electronmicroscope (Leica Cambridge Stereoscan 440 model).

\section{Results and discussion}

The incorporation of single, binary, ternary and quaternary systems of oligomeric HAS stabilizer, tertiary hindered amine, hindered phenol and organo-phosphite into LDPE was discussed by comparing the stabilizing efficiency of mixtures with and without phenol systems as well as with their counterparts of iPP and EP matrices.

\section{Tensile properties}

Figures 1 and 2 illustrate the changes in mechanical properties of neat and stabilized samples of LDPE before and after $\gamma$ sterilization with a dose of $25 \mathrm{kGy}$ where the stabilizing efficiency of phenol and phenol-free systems can be seen, in terms of elongation at break (\%), respectively. It is clearly seen that the elongation at break (\%) of neat sample was the lowest, while the samples with single, binary, ternary and quaternary stabilizer systems have shown higher values of elongation at break (\%). It can be understood from Figs. 1 and 2 that the order of efficiency of the stabilization in terms of tensile properties (i.e. protection against embrittlement) is as 


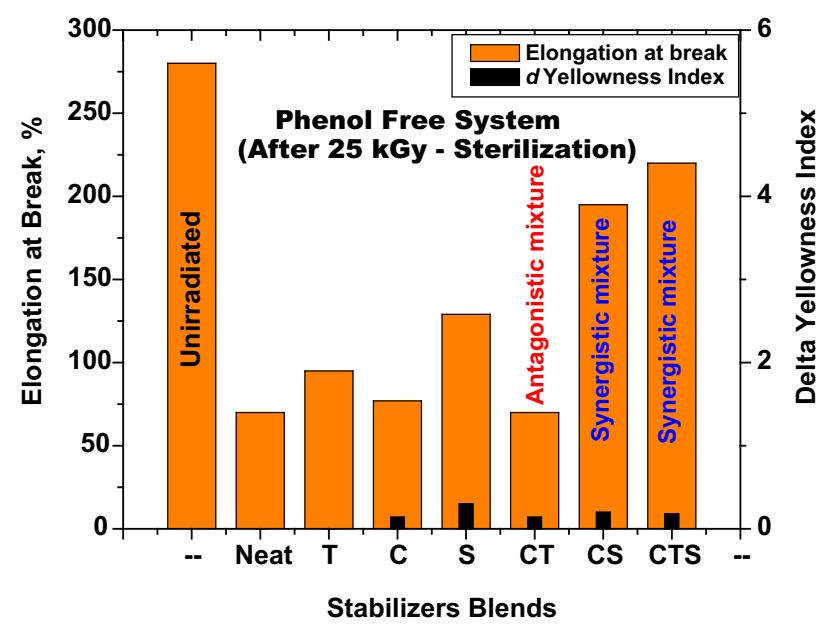

Fig. 1 Changes in tensile properties and yellow index of phenol-free system after $\gamma$-irradiation ( $25 \mathrm{kGy})$

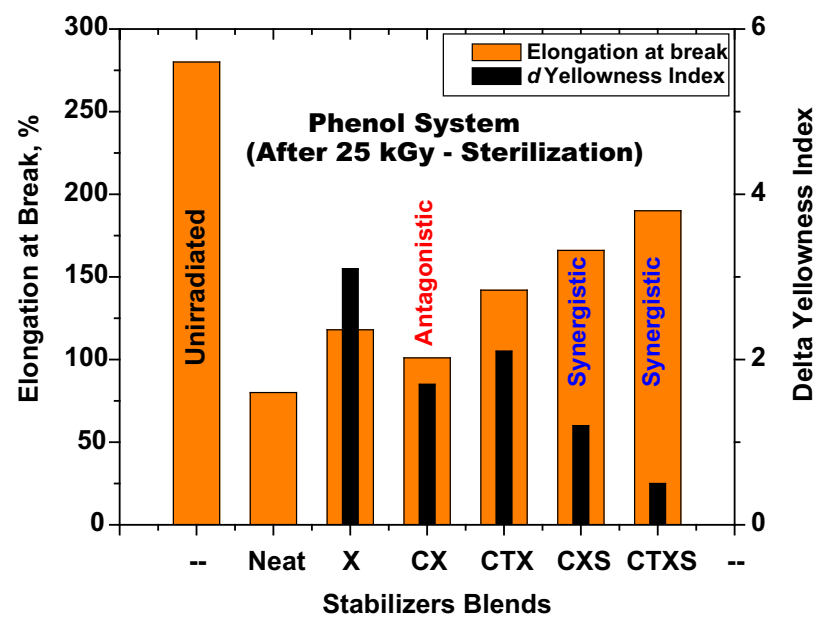

Fig. 2 Changes in tensile properties and yellow index of phenolic system after $\gamma$-irradiation ( $25 \mathrm{kGy}$ )

follows: $\quad$ CTS $>$ CS $>$ CTXS $>$ CXS $>$ CTX $>$ S $>$ X $>$ $\mathrm{CX}>\mathrm{T}>\mathrm{C}>\mathrm{CT}$. This indicates that the combination of various stabilizers with different molecular weights and protecting mechanisms significantly enhanced the efficiency of stabilization. In comparison with iPP \& EP matrices, the trend in stabilizing efficiency in terms of tensile properties is almost similar but the difference was observed only in the magnitude of the elongation at break (\%).

\section{Single-stabilizers systems}

In comparison of single-stabilizer systems, the samples containing organo-phosphite ' $S$ ' (Irgafos-168) have shown higher stabilization against $\gamma$-sterilization, while the samples stabilized with oligomeric HAS stabilizer ' $\mathrm{C}$ ' (Chimassorb-944) were the lowest and became the liable of embrittlement. The lower stabilization efficiency of the oligomeric stabilizer ' $\mathrm{C}$ ' may be ascribable to its high molecular weight and immobility in the polymer matrix [28]. Among the individual stabilizer system, HAS stabilizers [i.e. sec-HAS (Chimassorb-944) ' $\mathrm{C}$ ' and tert-HAS (Tinuvin-765) ' $\mathrm{T}$ '] have shown the lower protection against $\gamma$-degradation compared to hindered phenol (Irganox-1010) ' $\mathrm{X}$ ' and organo-phosphite (Irgafos-168) 'S'. The order of stabilization efficiency in the individual stabilizer systems in terms of tensile properties can be $\mathrm{S}>\mathrm{X}>\mathrm{T}>\mathrm{C}$.

\section{Phenol-free systems}

The changes in tensile properties of stabilized samples with phenol-free systems are revealed in Fig. 1. Among all stabilized samples, the reduction in elongation at break (\%) of 'CTS' samples was lesser than other samples. In case of binary systems, 'CS' have shown higher value of elongation than that of 'CT'. As observed in iPP and EP matrices, the sample CT in LDPE has shown to be more susceptible to breakage indicating that ' $\mathrm{CT}$ ' is an antagonistic mixture in the three polymer matrices. It is obvious that the combination of oligomeric HAS stabilizer ' $\mathrm{C}$ ' and tert-HAS ' $\mathrm{T}$ ' exhibits an antagonistic effect, while the combination of oligomeric HAS stabilizer and organo-phosphite exhibits synergistic effect. Similarly was found that the addition of organo-phosphite ' $\mathrm{S}$ ' to the antagonistic mixture (CT) has improved elongation at break (\%) considerably. The order of stabilization efficiency in the phenol-free systems is as follows: $\mathrm{CTS}>\mathrm{CS}>\mathrm{S}>\mathrm{T}>\mathrm{C}>\mathrm{CT}$.

\section{Phenol systems}

The changes in tensile properties of stabilized samples with phenol systems are illustrated in Fig. 2. Among the phenol systems, the samples 'CTXS' and 'CXS' were observed to be highly stabilized, while the sample 'CTX' have shown the higher decrease in elongation at break (\%). It is also obvious that the combination of ' $\mathrm{X}$ ' with the oligomeric HAS stabilizer ' $C$ ' [i.e. $C X$ ] shows the antagonistic effect of stabilization, while addition of ' $\mathrm{S}$ ' to ' $\mathrm{CX}$ ' and 'CTX' improves the polymer resistance against degradation. The phenol and phenol-free systems have shown increased stability against tensile breakage after $\gamma$-sterilization and it is supporting the trend observed in iPP and EP matrices [20-22]. For example, the elongation at break (\%) of antagonistic mixtures (i.e. CT and CX), 'CTX' and C was significantly improved stabilization efficiency after addition of organo-phosphite ' $\mathrm{S}$ '. However, the combination of hindered phenol ' $\mathrm{X}$ ' with ' $\mathrm{C} T$ ' increases the elongation at break (\%) as can be seen for the sample 'CTX'. It is clearly seen that the addition of ' $\mathrm{S}$ ' in the phenol and phenol-free systems exhibited synergistic effects. The results of tensile test has clearly shown that the stabilization efficiency of 
oligomeric HAS stabilizer [i.e. Chimassorb-944] can be improved by combinations with other stabilizers having different stabilization mechanisms and molecular weights.

\section{Yellowness index (YI)}

The color formation in neat and stabilized samples after $\gamma$ serialization is also represented in terms of delta yellowness index $(d$ YI) in Figs. 1 and 2. The effect addition of oligomeric HAS, tert-HAS and organo-phosphite to hindered phenol can be seen in Fig. 2 where yellowness index reduced. It can be seen that the combination of hindered phenol with other stabilizers highly prevent the discoloration. For example, the combination of hindered phenol with oligomeric HAS, tert-HAS and organo-phosphite (i.e. CTXS) drastically reduces the yellowness index from 3.1 to 0.5 . It can be seen that the addition of organo-phosphite (S) to $(\mathrm{CX})$ reduced the YI values from 1.9 to 1.1 , while addition of tert-HAS (T) to (CX) did not reduce the yellowness index. The significant reduction in yellowing after addition organo-phosphite (S) to CX and CTX can be attributed to the fact that organo-phosphite decomposes the hydroperoxides to reduce further oxidation and their reaction with oxidized products of hindered phenol, i.e. highly colored quinonoids which are transformed into colorless benzenoid forms [20-22, 34]. In contrast to tensile property, where the combination of oligomeric HAS stabilizer ' $C$ ' and hindered phenol ' $X$ ' (i.e. CX) exhibited the antagonistic effect, the incorporation of oligomeric HAS stabilizer into hindered phenol ' $\mathrm{X}$ ' significantly diminished the YI from 3.1 to 1.8. As explained [20-22], the reduction in YI after combination oligomeric HAS with hindered phenol may be due to the radical scavenging effect of oligomeric HAS stabilizer. The efficiency of phenolic stabilizer systems for protection against yellowing is as follows: CTXS $>$ CXS $>$ CX $>$ CTX $>$ X. This trend is similar to that observed for iPP and EP, but the magnitude of discoloration was lower in all samples of LDPE than that of iPP and EP due to the high durability of LDPE against $\gamma$ radiation. The results clearly indicate that the combination of hindered phenol with different stabilizers highly prevent discoloration. This could be explained by the presence of various stabilizers which prevent the polymer degradation via different protection mechanisms at different stages synergistically.

\section{Morphological aspects}

SEM is a reliable tool to monitor the surface changes during degradation of polymers. Figure 3 shows the scanning electron micrographs of neat and stabilized samples after $25 \mathrm{kGy} \gamma$-sterilization. It is evident from these micrographs that under $\gamma$-sterilization, neat sample was observed to show deformed/cracked surface (Fig. 3a). The crack formation on the surface of stabilized samples is lesser and samples 'S' (Fig. 3b) and 'CTS' (Fig. 3c) have shown mostly smooth surface. The higher surface erosion was observed in sample 'CT' (Fig. 3d) which shows the antagonistic effect confirming the result of elongation at break (\%). As seen in Figs. 1 and 2, the decrease in tensile properties of 'CT' sample also can be explained through the crack formation on the surface. Since eroded surface or cracks on the surface can act as 'defects' where failure mechanism is initiated, the tensile properties of the surface eroded samples can be lowered. The synergistic effect of organo-phosphite ' $S$ ' can be appreciated (by adding ' $S$ ' to 'CT') in 'CTS' which exhibited the higher stability against surface crack. The sample 'CTS' has shown stability against the crack formation than other samples. In comparison with iPP and EP matrices, the trend in stabilization efficiency is almost similar, but the surface of LDPE matrix was not much cracked as it was observed in iPP and EP matrix.

\section{IR spectroscopic analysis}

\section{Kinetics by carbonyl group evolution}

The order of stabilization efficiency can be confirmed further by determining the concentration of carbonyl group at the dose of $\gamma$-sterilization ( $25 \mathrm{kGy}$ ), while the stability period can be studied by monitoring the concentration of carbonyl group upon $\gamma$-irradiation with higher doses (above $25 \mathrm{kGy})$. The evolution of carbonyl group concentration $\left(\mathrm{mmol} \mathrm{L}^{-1}\right)$ upon $\gamma$-irradiation with different doses $(0-100 \mathrm{kGy})$ is plotted in Figs. 4 and 5. The concentration of carbonyl group increases with increasing the dose of $\gamma$ irradiation. It can be found in Fig. 4 that the rate of increase in concentration of carbonyl group of neat sample is higher than that of single-stabilizer systems with increasing irradiation dose. Up to sterilization dose, the order of efficiency of the stabilization in terms of preventing oxidation is as follows: $S>X>T>C$, i.e. the sample containing organo-phosphite ' $\mathrm{S}$ ' is more stabilized against oxidation than others. With increasing doses of $\gamma$ irradiation above sterilization dose $(25 \mathrm{kGy})$, this stabilization efficiency order is affected and for $100 \mathrm{kGy} \gamma$ irradiated samples, this order is changed as $\mathrm{X}>\mathrm{T}>\mathrm{C}>\mathrm{S}$. The changes in the efficiency order can be attributed to the fact that there may be the consumption of stabilizers during $\gamma$-irradiation up to higher doses. Figure 5 shows the carbonyl evolution in the phenol and phenol-free systems. It is obvious that the stabilization efficiency in the phenol and phenol-free systems is higher than that in single-stabilizer systems. In phenol-free systems, the higher stabilization efficiency in terms of 


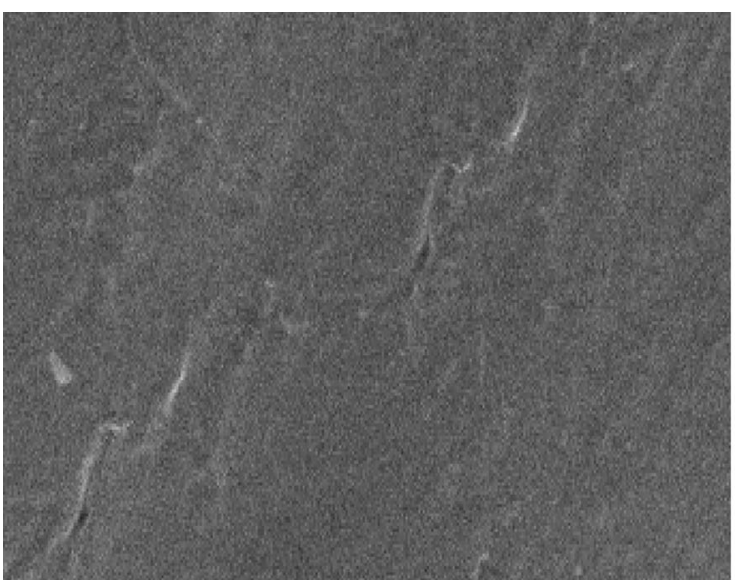

(a)

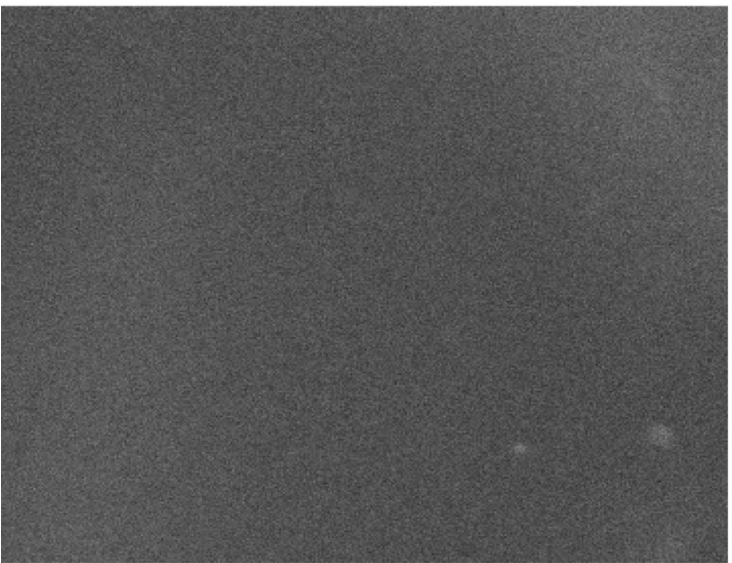

(c)

(Synergistic effect)

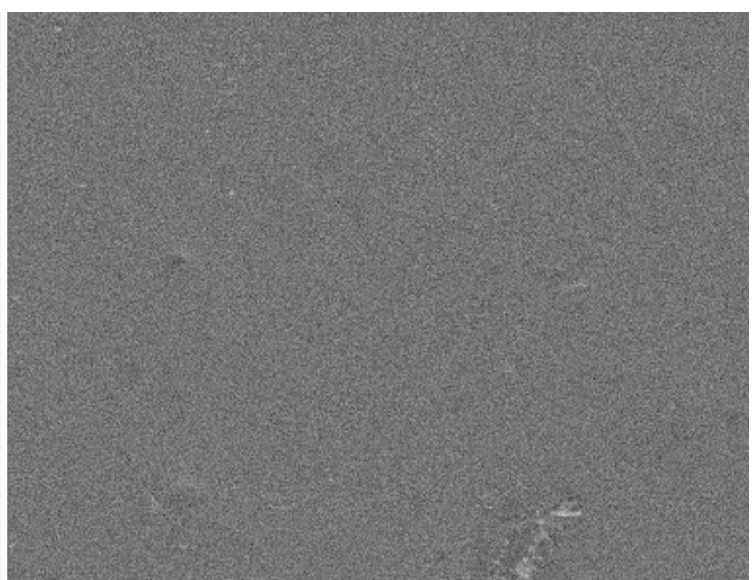

(b)

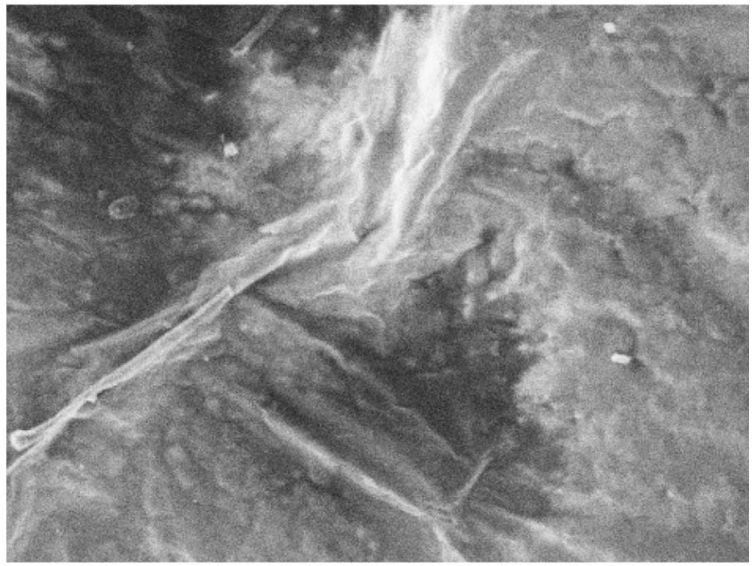

(d)

(Antagonistic effect)

Fig. 3 Scanning electron micrographs of $25 \mathrm{kGy} \gamma$-irradiated LDPE samples

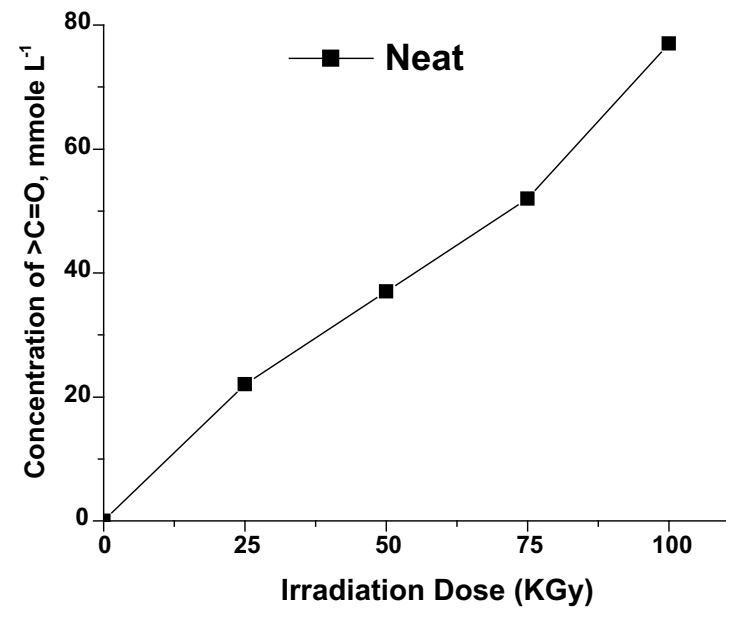

Fig. 4 Carbonyl group increase in neat and single-stabilizer systems

reduction in carbonyl group evolution was observed in CTS. However, the CT mixture has shown the antagonistic effects on oxidation with a linear increase in carbonyl group concentration (up to $100 \mathrm{kGy}$ ). An important

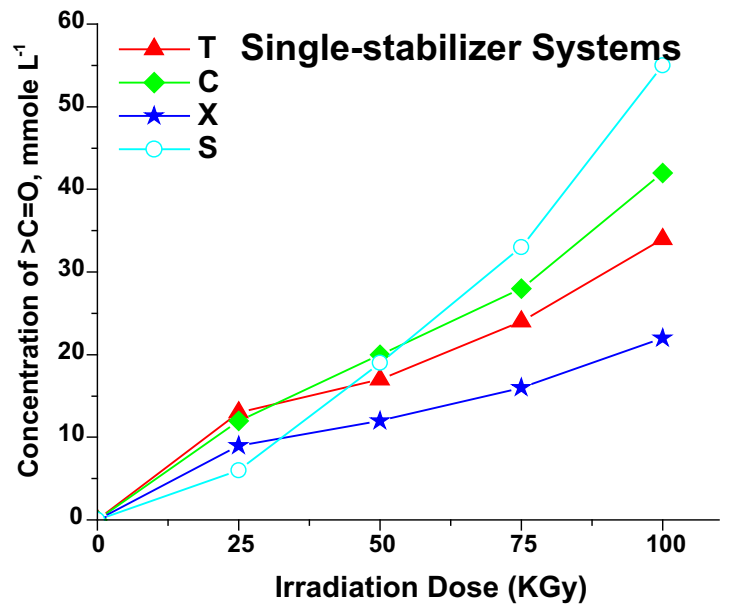

observation is that the kinetic accumulation of carbonyl group of CT mixture was a linear during irradiation up to $100 \mathrm{kGy}$, in accordance with the results of iPP and EP [20-22]. Similar to iPP and EP, it was also found that the 

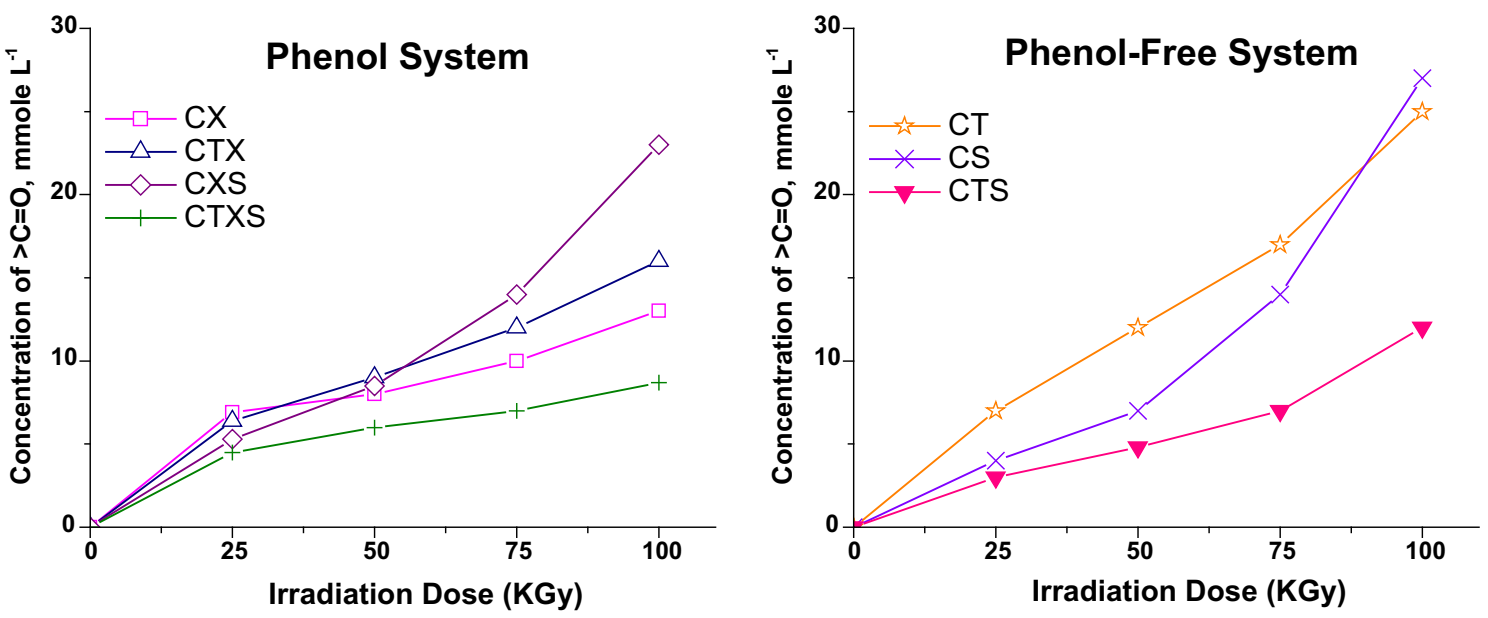

Fig. 5 Change in carbonyl group concentration of phenol and phenol-free systems upon $\gamma$-irradiation

stabilization efficiency in the phenol systems is higher than that in phenol-free systems. Accordingly, the addition of hindered phenol stabilizer $(\mathrm{X})$ to oligomeric mixtures $(\mathrm{CT}$, CTS, CS and C) lead to reduce the rate of oxidation during irradiation up to higher doses. It is evidence that the phenol systems exhibited longer period of stabilization against $\gamma$ irradiation for iPP, EP and LDPE. Among the phenol systems, the samples of binary (CX) and quaternary mixtures (CTXS) have shown higher stability. Among all the mixtures, CTXS and CTS have shown higher stability against all the doses of $\gamma$-irradiation indicating the longer durability for longer period. Up to $100 \mathrm{kGy} \gamma$-irradiation, the samples containing organo-phosphite became lower stabilized against oxidation, while samples containing hindered phenol showed higher stability. The samples containing organo-phosphite (i.e. S, CS and CXS) have shown low rates of carbonyl group concentration below $25 \mathrm{kGy}$ and thereafter suddenly increased indicating the short stabilization period.

The change in the order of stabilization efficiency, in terms of reduction in the rate of carbonyl group evolution, can be attributed to the consumption of stabilizers. This may be in the form of physical loss of stabilizer from the samples and/or transformations of active form stabilizer to inactive form during $\gamma$-irradiation. It was argued that if one stabilizer is not affecting the stabilization mechanism of other stabilizer, the rate of carbonyl group evolution should not be changed or the order of stabilization efficiency of mixtures should not be altered. As it was observed in Fig. 4 for the sample of ' $\mathrm{S}$ ', sudden increase in the carbonyl group above $\gamma$-irradiation dose of $25 \mathrm{KGy}$ can be attributed to the fact of the disappearance of Irgafos-168. This observation was already reported by Kawamura et al. [35] that Irgafos168 was fastest to disappear from the samples. These results are in agreement with those of Stoffer [36] who found that the amount of Irgafos-168 from polyolefins decreased with higher irradiation doses. Carlsson et al. [37] observed a complete degradation of phosphite to give mainly phosphate, but at quite low $\gamma$-irradiation doses ( $\sim 5 \mathrm{kGy}$ ) during $\gamma$-sterilization of HDPE trays. They also detected that any residual phosphite is lost progressively in post-irradiation reactions. Allen et al. [38] also reported that both gamma and electron beam irradiation had similar effects with regard to the extent to which Irgafos-168 was not detected after the dose of $25 \mathrm{kGy}$. Likewise, it was observed in Figs. 4 and 5 that in the samples containing organo-phosphite (S, CS and CXS) the rate of carbonyl group concentration suddenly increased above $25 \mathrm{kGy}$. The sudden increase in carbonyl group and the fast consumption of ' $S$ ' and its mixtures (i.e. CS and CXS) above $25 \mathrm{kGy}$ were also seen in the iPP and EP matrices. On the other hand, for the hindered phenol ' $\mathrm{X}$ ' sample, such increase in carbonyl group concentration was not observed, indicating that consumption of hindered phenol ' $\mathrm{X}$ ' is slower than others and/or lower rate of loss of hindered phenol is observed in the film. In mixture systems also, the samples containing ' $\mathrm{X}$ ' have shown higher protection ability at higher irradiation doses, especially in 'CTXS' and ' $C X$ ' samples, indicating the synergistic interaction of oligomeric HAS and hindered phenol by forming the compounds which are in turn radical scavengers [39, 40]. The retained concentration of oligomeric HAS stabilizer (Chimassorb-994) in all mixtures which determined from the triazine absorption [41] will reveal the contribution of added stabilizers in polymer matrix. Table 3 shows the retained concentration of oligomeric HAS stabilizer in all samples after sterilization with $25 \mathrm{kGy}$. It can be seen that the consumption of oligomeric HAS stabilizer was higher $(43 \% \mathrm{w} / \mathrm{w})$ when used in combination with ' $\mathrm{T}$ ' (i.e. CT) indicating the antagonistic effect of the CT mixture. However, the retained concentration was higher $(97 \% \mathrm{w} / \mathrm{w})$ when it is combined with ' $S$ ' (i.e. CS). The preservation of 
Table 3 The retained concentration of oligomeric HALS (Chimassorb 994) after $\gamma$-sterilization (25 kGy)

\begin{tabular}{lllllllll}
\hline Components & C & CT & CX & CS & CTX & CTS & CXS & CTXS \\
\hline Chimassorb 994 (C), retained (\%) & 80 & 43 & 71 & 97 & na & 92 & 87 & na \\
\hline
\end{tabular}

na not available

oligomeric HAS stabilizer was also observed whenever it combined with ' $\mathrm{S}$ ' (i.e. CS, CTS and CXS), which is an indication of the synergistic effect. Norman Allen [42] has found a synergistic effect in the heat and light degradation of PP and PE films when he examined the reaction between two phosphite stabilizers and universal polymeric hindered piperidine ultraviolet light stabilizers. This is because the phosphite stabilizers could destroy hydroperoxide and remove oxygen, thus protecting the Chimassorb-944, which may reflect why the retained concentration of oligomeric HAS (Chimassorb-994) in CS is higher (97\% w/w) after $\gamma$-sterilization. In comparison, the retained concentration of Chimassorb-994 was higher in the LDPE matrix than that in iPP and EP matrices.

In comparison with iPP and EP copolymers matrices, it can be found that the trend in stabilizing efficiency against embrittlement, discoloration, surface crack and oxidation is almost similar but the difference was observed only in the magnitude. In case of single-stabilizer systems, it was observed that the samples stabilized with high-molecularweight stabilizer (i.e. oligomeric HAS stabilizer, MW $2790 \mathrm{~g} / \mathrm{mol}$ ) and with relatively small-molecular-weight stabilizer [i.e. tert-HAS (Tinuvin-765) MW $508 \mathrm{~g} / \mathrm{mol}$ ] have shown the lower protection against $\gamma$-degradation. However, the samples stabilized with moderate-molecularweight stabilizers [Irgafos-168, MW $649.9 \mathrm{~g} / \mathrm{mol}$ and Irganox-1010, MW $1178 \mathrm{~g} / \mathrm{mol}$ ] have shown higher stability in terms of preventing embrittlement, surface erosion and oxidation. The lower stabilization efficiency of the high-molecular-weight stabilizer (oligomeric HAS) may be attributed to its immobility in the polymer matrix. However, the lower stabilization efficiency of the tert-HAS may be attributed to the physical loss due to its low molecular weight. It is well known that if a stabilizer molecule is too small, not only it will be incompatible with certain polymeric materials, but it probably diffuses and volatilize away from the polymer. This problem has been associated with relatively small-molecular-weight compounds such as Tinuvin-770 (MW 478). It was confirmed that the consumption of organo-phosphite (Irgafos-168) is faster in iPP, EP and LDPE matrices than other stabilizers. In case of binary, ternary and quaternary stabilizer systems, it was observed that the mixtures of oligomeric HAS and tertHAS (i.e. CT) and hindered phenol (i.e. CX) shows the antagonistic effect. However, the addition of organophosphite ' $\mathrm{S}$ ' in the phenol and phenol-free systems $(\mathrm{C}$,
CT, CX and CTX) exhibited synergistic effects of stabilization against embrittlement, discoloration, surface erosion and oxidation. Likewise, the consumption of oligomeric HAS stabilizer was found to be higher when it is used in combination with ' $\mathrm{T}$ ' and with ' $\mathrm{X}$ ' (i.e. $\mathrm{CT}, \mathrm{CX}$ ) indicating the antagonistic effect of CT and CX mixtures. However, the retained concentration of oligomeric HAS stabilizer was highly preserved whenever it combined with ' $S$ ' (i.e. CS, CTS and CXS), which is an indication of the synergistic effect. The synergistic effect obtained by combination of organo-phosphite with hindered phenols and hindered amines could be explained by the fact that organo-phosphite preserves the concentration of hindered phenols and replaces the hindered amines during polymer processing [43]. The phenol systems have shown longer period of stabilization than phenol-free systems. The longest period of stabilization was explained through the interaction between hindered phenol and hindered amine [20-22, 44-48]. Our experimental results demonstrated that the synergism and antagonism of the binary, ternary and quaternary stabilizer systems were almost similar in LDPE, iPP and EP matrices indicating that the interaction/ mechanisms of stabilizers are same, but LDPE was highly stabilized than iPP and EP matrices. The results of our research series proved that the synergistic mixtures based on oligomeric HAS stabilizer (i.e. CS, CTS, CTX, CXS, CTXS) significantly improve the stability of polyolefins (LDPE, iPP and EP copolymers) against $\gamma$-sterilization. The results also demonstrated that the phenol-free systems (i.e. CTS) can be good alternatives for the phenolic systems (i.e. X, CTX, CXS and CTXS).

The main objective of our research series was to find out the various possibilities of combinations of stabilizers which follow the different mechanisms of stabilization and are already approved for food contact and biomedical polyolefin applications. The objective of the present work is to study the synergism and antagonism and the stabilizing efficiency of those systems with LDPE and to conduct a comparative study on their effectiveness with other polyolefins matrices (iPP and EP copolymers). In iPP and EP matrices, possibilities of various binary, ternary and quaternary additive systems have been found [20-22]. Potentials of those systems in LDPE matrix were tested here. Almost the trend in stabilization efficiency is the same. The trend in stabilization efficiency of binary, ternary and quaternary systems of hindered amines, hindered 
phenol and organo-phosphite was confirmed in terms of tensile properties, discoloration and oxidation products as observed for iPP and EP copolymers. Thus, the mechanisms of stabilization of each stabilizer are not disturbed by various polyolefins. The obtained data may reflect the suitability, selection of different kinds of stabilizers and antioxidants to be combined with oligomeric HAS stabilizer and its effectiveness. The data also reveal the stabilization behavior of oligomeric HAS stabilizer when it was used alone and when it is used in combination with different stabilizers for protecting different polyolefins (LDPE, iPP and EP copolymers) against $\gamma$-sterilization. The results of the present study confirm the validity of those systems for protecting various polyolefins (LDPE, iPP and EP copolymer) against $\gamma$-sterilization using synergistic mixtures of stabilizers, which follow the different mechanisms of stabilization and are already approved for food contact applications.

\section{Conclusion}

The stabilization of $\gamma$-sterilized LDPE was tested with the mixtures of different stabilizers, which follow the different stabilization mechanisms and are having various molecular weights and are approved for food contact applications. The major objective of this study ws to conduct a comparative study on the effectiveness of those mixtures on LDPE with other polyolefin matrices (iPP and EP copolymers). In this study, we found that the synergism, antagonism and the trend in stabilization efficiency of the binary, ternary and quaternary stabilizer systems of oligomeric HAS, tertiary hindered amine, hindered phenol and organo-phosphite were almost similar in LDPE, iPP and EP copolymer matrices indicating that the interaction/mechanisms of stabilizers are same. The results show that the polyolefin's durability and yellowing formation due to phenolic antioxidants can be improved significantly by adding oligomeric HAS, tert-HAS and organo-phosphite, leading also to long-term stability. The molecular weight distribution of stabilizers in the mixture plays an important role in the overall stability. It was demonstrated that the phenol-free systems can be suitable alternatives for the phenolic systems. Thus, it can be concluded from the results of our research series that the stability of food packaging and medical polyolefins (LDPE, iPP and EP copolymers) against $\gamma$-sterilization can be improved by blends of different stabilizers which are approved for food contact applications.

Acknowledgements The authors are grateful to the director and the scientists in National Chemical Laboratory, Pune, council of Scientific and Industrial Research (CSIR), New Delhi, India and to Prof.
B. S. M. Rao, Department of Chemistry, University of Pune, Pune, India for providing the facilities during this work.

Open Access This article is distributed under the terms of the Creative Commons Attribution 4.0 International License (http://crea tivecommons.org/licenses/by/4.0/), which permits unrestricted use, distribution, and reproduction in any medium, provided you give appropriate credit to the original author(s) and the source, provide a link to the Creative Commons license, and indicate if changes were made.

\section{References}

1. Booth AF (1979) Sterilization of Medical devices. Interpharm Press, Illinois, Buffalo Grove

2. Sturdevant M (1991) Plast Eng 47:27-32

3. Deng M, Shalaby SW (1995) Effects of gamma irradiation, gas environments, and post-irradiation aging on ultrahigh molecular weight polyethylene. J Appl Polym Sci 58:2111-2119

4. Goldman M, Lee M, Grousky R, Pruitt L (1997) Oxidation of ultrahigh molecular weight polyethylene characterized by Fourier Transform Infrared Spectrometry. J Biomed Mater Res 37(1):43-50

5. Fisher J, Reeves EA, Isaac GH, Saum KA, Sanford WA (1997) Comparison of the wear of aged and non-aged ultrahigh molecular weight polyethylene sterilized by gamma irradiation and by gas plasma. J Mater Sci-Mater Med 8(6):375-378

6. Shintani H (2002) Effects of ionizing radiation sterilization treatment on medical use plastic materials. Biocontrol Sci $7(1): 1-8$

7. Klemchuk PP, Horng PL (1991) Transformation products of hindered phenolic antioxidants and colour development in polyolefins. Polym Degrad Stab 34:333-346

8. Emaldi I, Liauw CM, Potgieter H (2015) Stabilization of polypropylene for rotational molding applications. J Res Updates Polym Sci 4:179-187

9. Carlsson DJ, Chmela S (1983) Polymers and high-energy irradiation: degradation and stabilization. In: Jellinek HHG (ed) Degradation and stabilization of polymers, vol 1, chap 4. Amsterdam, Elsevier 1983

10. Nasibullah M, Ahmad N, Hassan F, Patel DK, Khan AR, Masihur R (2012) Assessment of physicochemical parameters of tubing's of intravenous infusion sets. Res J Pharm Sci 1(4):1-9

11. Nasibullah M, Ahmad N, Patel DK, Masihur R (2012) Assessment of Physicochemical Parameters in Nasal Feeding Tubes. Int J Life Sci Pharma Res 2(3):20-24

12. Vedanarayanan PV, Fernandez AC (1987) Toxicology of Biomedical Polymers. Def Sci J 37(2):173-183

13. Scott G (1995) Antioxidants in food packaging: a risk factor? Biochem SOC Symp 61:235-246 (printed in Great Britain)

14. David F (1981) In: Williams (ed) Systemic Aspects of Biocompatibility, vol 11. CRC-Press

15. Billingham N (1990) In: Pospisil J, Klemchuk PP (eds) Oxidation Inhibition in Organic Materials, chap 6:2. CRC Press, Boca Raton

16. Al-Malaika S, Goonetilleka MRJ, Scott G (1991) Migration of 4-substituted 2-hydroxy benzophenones in low density polyethylene: Part I - Diffusion characteristics. Polym Degrad Stab 32:231-247

17. Hassanpour S, Khoylou F (2007) Synergistic effect of combination of Irganox 1010 and zinc stearate on thermal stabilization of electron beam irradiated HDPE/EVA both in hot water and oven. Radiat Phys Chem 76:1671-1675

18. Haidar N, Karlsson S (2001) Loss of Chimassorb 944 from LDPE and identification of additive degradation products after exposure to water, air and compost. Polym Degrad Stab 74:103-112 
19. Malik J, Hrivik A, Alexyova D (1992) Polym Degrad Stab 35:25

20. Alariqi SAS, Pratheep Kumar A, Tevtia AK, Rao BSM, Singh RP (2006) Stabilization of $\gamma$-sterilized biomedical polyolefins by synergistic mixtures of oligomeric stabilizers. Polym Degrad Stab 91:2451-2464

21. Alariqi SAS, Kumar AP, Rao BSM, Singh RP (2007) Stabilization of $\gamma$-sterilized biomedical polyolefins by synergistic mixtures of oligomeric stabilizers. Part II. Polypropylene matrix. Polym Degrad Stab 92:299-309

22. Alariqi SAS (2015) Stabilization of $\gamma$-sterilized food-packaging materials by synergistic mixtures of food-contact approval stabilizers. Int J Biol Food Vet Agric Eng 9(2)

23. Przybytniak G, Mirkowski K, Rafalski A, Nowicki A, Legocka I, Zimek Z (2005) Effect of hindered amine light stabilizers on the resistance of polypropylene towards ionizing radiation. NUKLEONIKA 50(4):153-159

24. Sun GJ, Jang HJ, Kaang S, Chae KH (2002) A new polymeric HALS: preparation of an addition polymer of DGEBA-HALS and its photostabilizing effect. Polymer 43:5855-5863

25. Malik J, Hrivik A, Tomova E (1992) Diffusion of hindered amine light stabilizers in low density polyethylene and isotactic polypropylene. Polym Degrad Stab 35:61-66

26. Kikkawa K (1995) New developments in polymer photostabilization. Polym Degrad Stab 49:135-143

27. Chmela S, Hrdlovic P, Manasek Z (1985) Polym Degrad Stab 11:233

28. Clough L, Billingham NC, Gillen KT (1996) Polymer durability degradation, stabilization, and lifetime prediction. American Chemical Society, Washington, DC, pp 455-470

29. Gugumus F (1981) Res Discl 209:357

30. Ciba specialty chemicals additives for polyolefins key products selection guide, Pub. No. 016269.00.040, Switzerland: Ciba Specialty Chemicals Inc. http://cibasc.com/

31. Carlsson DJ, Wiles DM (1969) The photodegradation of polypropylene films. II. photolysis of ketonic oxidation products. Macromolecules 2:587-597

32. Annual Book of ASTM Standard (1988) American Society for Testing and Materials, vol 8.02, Philadelphia

33. Billmeyer FW, Sultzman (1966) Principles of colour Technol. Interscience, New York, p 38

34. Clough L, Billingham NC, Gillen KT (1996) Polymer durability degradation, stabilization, and lifetime prediction, chap 39 . American Chemical Society, Washington, DC, pp 375-396

35. Kawamura Y, Sayama K, Yamada T (2000) Shokuhin Shosha 35(1-2):7-14
36. Stoffer NH (2004) Certified reference materials for food packaging specific migration tests: development, validation and modeling. $\mathrm{Ph} . \mathrm{D}$. Thesis, University Wageningen, The Netherlands

37. Carlsson DJ, Krzymien ME, Deschenes L, Mercier M, Vachon C (2001) Phosphite additives and their transformation products in polyethylene packaging for $\gamma$-irradiation. Food Addit Contam 18(6):581-591

38. Allen DW, Leathard DA, Smith C (1991) The effects of gammairradiation on the fate of hindered phenol antioxidants in food contact polymers - analytical and C-14 labeling studies. Radiat Phys Chem 38(5):461-465

39. Pospisil J (1993) Chemical and photochemical behaviour of phenolic antioxidants in polymer stabilization: a state of the art report, part II. Polym Degrad Stab 39:103-115

40. Vyprachticky D, Pospisil J (1990) Possibilities for cooperation in stabilizer systems containing a hindered piperidine and a phenolic antioxidant-a review. Polym Degrad Stab 27(3):227-255

41. McFarlane D (2002) Diffusion controlled investigation of the parameters responsible for the efficiency of hindered amine light stabilizers, Ph.D. Thesis, Department of Applied Chemistry, Faculty of Applied Science, Royal Melbourne Institute of Technology, Melbourne, Australia

42. Allen NS, Ortiz RA, Anderson GJ (1998) Interaction in the thermal and light stabilizing action of novel aromatic phosphites with a 2-hydroxybenzophenone and hindered piperidine stabilizer in polyolefin film. Polym Degrad Stab 61(2):183-199

43. Murayama KJ (1971) Synth Org Chem 29:366

44. Pospisil J (1991) The key role of antioxidant transformation products in the stabilization mechanisms - a critical analysis. Polym Degrad Stab 34:85-109

45. Pospisil J (1992) Exploitation of the current knowledge of antioxidant mechanisms for efficient polymer stabilization. Polym Adv Technol 3:443-455

46. Carloni P, Greci L, Stipa P, Rizzoli C, Sgatabotto P, Ugozzoli F (1993) Antioxidants and light stabilizers. Part 1. Reactions of an indolinone nitroxide and phenoxy radicals. X-ray crystallographic analysis of 1-[O-(3,5-di-tert-butyl-4-hydroxy)-benzyl]-1,2-dihydro-2-methyl-2-phenyl-3-oxo-3H-indole and 3,5,3'5'-tetra-tertbutylstilbene-4,4'-quinone. Polym Degrad Stab 39:73-83

47. Pauquet JR (1992) In: Processing of wire and cable, the 42nd international symposium. Eatontown, NJ, pp 35:105

48. Drake WO, Pauquet JR, Tedesco RV, Zweifel H (1990) Processing stabilization of polyolefins. Angew Makromol Chem 176(177):215-230 